\title{
CONTRIBUCIÓN AL CONOCIMIENTO MELITOPALINOLÓGICO DE HUELVA Y SEVILLA
}

\author{
Pedro L. ORTIZ e Inmaculada FERNÁNDEZ
}

\begin{abstract}
RESUMEN. Contribución al conocimiento melitopalinológico de Huelva y Sevilla. Se ha estudiado al microscopio óptico el sedimento de 19 muestras de miel producidas en primavera en varias localidades de las comarcas del Andévalo (Huelva) y la Sierra Norte de Sevilla. Los resultados obtenidos se comparan con los de anteriores estudios de mieles de estas comarcas producidas durante todo el año (cosecha de apicultores). Se pone de relieve que la mielada tiene poco significado como fuente de miel durante la primavera, aunque no es así en otros periodos del año. Los resultados disponibles sugieren que, en su conjunto, la flora de interés apícola de la Sierra Norte de Sevilla es más diversa que la del Andévalo, aunque ello no se traduce en que las mieles del Andévalo individualmente tengan espectros polínicos con menor número de tipos. La tercera parte de los tipos polínicos encontrados en este trabajo no se habían encontrado con anterioridad en muestras de estas comarcas, sin embargo los principales recursos apícolas ya se habían detectado antes. Los resultados actuales destacan notablemente el papel de Echium plantagineum y de Lavandula stoechas s.l. como recursos nectaríferos de primavera y señalan la posibilidad de obtención de mieles monoflorales de estas especies. Asimismo queda destacado el papel de varias especies de Cistus como principales recursos poliníferos primaverales. Entre las especies cuyo polen se ha encontrado por primera vez en estas comarcas destacan Teucrium capitatum como fuente de néctar y Cistus libanotis de polen, ambos con interés local.
\end{abstract}

Palabras clave. Palinología, melitopalinología, miel, polen, recursos naturales, Huelva, Sevilla, Andalucía.

ABSTRACT. Contribution to melissopalynological knowledge of Huelva and Sevilla. Nineteen spring honey samples from different localities of the regions of El Andévalo (Huelva) and North Sierra of Sevilla have been studied by light microscopy. The results are compared with those of previous melissopalynological studies of these regions. It is shown that honeydew is little important as honey source during spring, though it is not the same in other seasons. Available data suggest that, as a whole, the apiculturally-interesting flora of North Sierra of Sevilla is more diverse than that of El Andévalo, but pollen spectra of El Andévalo honeys, individually, have not a lesser number of types. One-third of the pollen types found in this work had not been recorded before in samples from these regions, but the main apicultural resources had been already detected in the previous studies. The current results emphasize notably the role of Echium plantagineum and Lavandula stoechas as spring nectar sources, and point out the possibility of getting unifloral honeys from these taxa. In like manner, it is emphasized the role of several species of Cistus as main pollen resources during spring. Among the taxa whose pollen grains have been found in this work for the first time in samples from these regions, Teucrium capitatum, as nectar source, and Cistus libanotis, as pollen source, are the most outstanding ones, their importance being local.

Key words. Palynology, melissopalynology, honey, pollen, natural resources, Huelva, Sevilla, Andalucía. 


\section{INTRODUCCIÓN}

Es un hecho conocido que el análisis microscópico de la miel aporta información tanto sobre las preferencias alimenticias de sus productoras como sobre su origen botánico (Louveaux et al., 1978; Maurizio, 1979; Ortiz, 1991).

En los últimos años se han realizado numerosos trabajos melitopalinológicos, algunos de ellos en las provincias de Huelva y Sevilla. La comarca onubense de Aracena ha sido objeto de dos de estos estudios: Tello (1982) y Ortiz et al. (1990); las mieles de Doñana y de la comarca de El Andévalo, también en Huelva, han sido estudiadas por Gómez y Sáenz (1985) y Ortiz (1988), respectivamente; Ortiz y Fernández (1992) analizaron mieles de toda la provincia de Sevilla; asimismo, el trabajo de Serra y Cañas (1988) sobre mieles monoflorales de eucalipto incluye muestras de Huelva y Sevilla, sin especificar localidades concretas de procedencia.

En el presente trabajo se estudian muestras procedentes de El Andévalo y la Sierra Norte de Sevilla. En los estudios melitopalinológicos realizados previamente en estas comarcas (Ortiz, 1988; Ortiz y Fernández, 1992) se han analizado muestras de miel de las cosechas de los apicultores, que efectúan una sola cata anual; estos trabajos han puesto de manifiesto cuáles son las principales fuentes de miel y polen para las abejas en el conjunto del año: en ambas comarcas Eucalyptus sp., Echium plantagineum y Lavandula stoechas s.l. son recursos melíferos importantes, habiendo sido caracterizadas mieles monoflorales de eucalipto en El Andévalo, y Cistus sp., Quercus sp., Eucalyptus sp. Echium plantagineum y Myrtus communis son los recursos polínicos más destacables. Además, es más que probable que algunas de las mieles de Eucalyptus caracterizadas por Serra y Cañas (1988) procedan de estas comarcas. Sin embargo, es bien sabido que en Andalucía Occidental la floración presenta una acusada estacionalidad, y en general se concentra en primavera; en áreas vecinas a las comarcas objeto de este estudio, la mayor concentración de especies en flor ha sido observada durante el mes de Mayo (Arroyo, 1988, 1990; Talavera et al., 1988; Ortiz, 1991). Por este motivo, hemos considerado que el estudio de nuevas muestras de miel de estas comarcas producidas durante ese periodo de máxima floración puede ampliar la información disponible sobre los recursos apícolas de estas comarcas. El interés de plantear este trabajo aumenta si se tiene en cuenta que el número de muestras estudiadas en los trabajos efectuados anteriormente en estas comarcas no es elevado, sobre todo en el caso del Andévalo.

\section{ÁREA DE ESTUDIO}

La comarca del Andévalo se extiende por el SO de la provincia de Huelva, y está constituida por sierras pizarrosas con altitudes inferiores a $500 \mathrm{~m}$. Es una región muy degradada donde apenas quedan vestigios del bosque potencial (encinar o alcornocal), pero existen algunas dehesas; son muy abundantes en cambio, los matorrales de sustitución, generalmente jarales-aulagares o a veces brezales en los enclaves más húmedos. Además, existen enormes extensiones de repoblaciones con Eucalyptus camaldulensis y E. globulus.

La Sierra Norte de Sevilla es una comarca montañosa con elevaciones de hasta $900 \mathrm{~m}$ y compuesta principalmente de materiales graníticos y pizarrosos. La vegetación boscosa se encuentra bien conservada en algunos enclaves, pero en general está bastante degradada, existiendo extensas áreas adehesadas y vastos matorrales, generalmente jarales y algunos brezales. Asimismo, hay amplias plantaciones de eucaliptos.

Para una descripción más exhaustiva de la 
vegetación y la corología en el área de estudio puede consultarse Ladero (1987) y Valdés et al. (1987b).

\section{MATERIAL Y MÉTODOS}

Se han estudiado muestras de miel procedentes de tres colmenares del Andévalo, uno situado en Niebla y dos en Alosno, y de dos colmenares de la Sierra Norte de Sevilla, uno en El Ronquillo y otro en El Pedroso (en ambos casos las colmenas eran tipo Layens). Los cinco colmenares se encontraban en áreas de jaral, en cuyas proximidades había eucaliptos y alguna dehesa o algún vestigio de bosque.

En cada colmenar se han seleccionado entre 3 y 5 colmenas sanas y bien pobladas, y de cada una de ellas se ha obtenido una muestra de miel. Para ello, en cada colmena se colocó un cuadro con cera sin estirar en la segunda quincena de Abril de 1987 y se retiró en los primeros días de Junio siguiente; para entonces las abejas ya habían construido las celdillas y almacenado provisiones. De cada cuadro se han cortado aquellos trozos de panal que sólo contenian miel; cualquier resto de cargas de polen encontrado en estos fragmentos de panal ha sido eliminado cuidadosamente, para evitar añadírselo a la miel; los trozos de panal de cada cuadro han sido prensados manualmente para obtener una muestra de su miel.

El sedimento de estas muestras se ha examinado microscópicamente para identificar y contar sus constituyentes botánicos. El número absoluto de granos de polen y de elementos de mielada se ha determinado sobre preparaciones elaboradas sin ningún tratamiento químico, siguiendo básicamente a Maurizio (1979). En cambio, se ha usado el método acetolítico de Erdtman (1960), ligeramente modificado, para elaborar las preparaciones sobre las que se han identificado los tipos polínicos y se han determinado sus frecuencias. Los procesos de elaboración de ambos tipos de preparaciones han sido descritos detalladamente en Ortiz (1991). Siguiendo las indicaciones de Vergeron (1964) y Louveaux et al. (1978), se han contado e identificado al menos 1200 granos de polen repartidos en cuatro preparaciones diferentes de cada muestra. Para la identificación de los tipos polínicos se han usado la clave palinológica de Valdés et al. (1987a) y las preparaciones de referencia de la Palinoteca del Departamento de Biología Vegetal y Ecología de la Universidad de Sevilla. Además, se ha tenido en cuenta el área de distribución de cada especie (Valdés et al., 1987b) y la experiencia personal en el campo. Siempre que ha sido posible se ha identificado el tipo polínico a nivel de especie; en caso contrario se ha llegado a nivel genérico o de tipo polínico que incluye géneros afines.

\begin{tabular}{lrrr}
\hline MUESTRAS & NGP & NEM & CLASE \\
\hline Niebla 1 & 26400 & 900 & II \\
Niebla 2 & 76500 & 2850 & II \\
Niebla 3 & 27250 & 1050 & II \\
Niebla 4 & 29850 & 2150 & II \\
Alosno A1 & 94200 & 4000 & II \\
Alosno A2 & 24800 & 1650 & II \\
Alosno A3 & 58550 & 1750 & II \\
Alosno A4 & 20050 & 3700 & II \\
Alosno A5 & 60450 & 2150 & II \\
Alosno B1 & 53050 & 5050 & II \\
Alosno B2 & 58050 & 10650 & II \\
Alosno B3 & 26350 & 4650 & II \\
Alosno B4 & 41850 & 4050 & II \\
El Ronquillo 1 & 152200 & 4150 & III \\
El Ronquillo 2 & 57450 & 6800 & II \\
El Ronquillo 3 & 245850 & 7600 & III \\
El Pedroso 1 & 66950 & 2050 & II \\
El Pedroso 2 & 172900 & 3150 & III \\
El Pedroso 3 & 80400 & 5650 & II \\
\hline
\end{tabular}

Tabla 1.- Número de granos de polen (NGP) y de elementos de mielada (NEM) contenidos en 10 gramos de miel. Las clases corresponden a las establecidas por Maurizio (1949) sec. MAURIZIO (1979). Number of pollen grains (NGP) and number of honeydew elements (NEM) contained in 10 grammes of honey. The classes are those established by Maurizio (1949) sec. MAURIZIO (1979). 


\section{RESULTADOS}

En la tabla 1 aparecen el número de granos de polen y de elementos de mielada contenidos en 10 gramos de cada muestra. En general el sedimento de las mieles estudiadas no es abundante, predominando entre ellas las pertenecientes a la clase II de Maurizio (ver Maurizio, 1979). Dicho sedimento está constituido en todos los casos tanto por granos de polen como por elementos indicadores de mielada. Al igual que en las muestras de estas comarcas estudiadas con anterioridad (Ortiz, 1988; Ortiz y Fernández, 1992), los elementos de mielada aquí encontrados son hifas y esporas de hongos.

En las muestras estudiadas se han identificado 39 tipos polínicos presentes en alguna de ellas al menos en un $1 \%$. En la tabla 2 se detallan estos tipos y sus frecuencias de aparición en cada una de las mieles estudiadas. En las muestras del Andévalo están representados 30 de ellos, mientras que en las de la Sierra Norte de Sevilla sólo aparecen 23. Más de un tercio de los tipos identificados en este trabajo no habían sido detectados en las muestras de estas comarcas estudiadas anteriormente (Ortiz, 1988; Ortiz y Fernández, 1992), pero su representación es escasa en casi todos los casos (tabla 2).

El número de tipos con frecuencia mayor del $1 \%$ por muestra oscila entre 4 y 17 ( $\overline{\mathrm{x}} \pm \mathrm{E} . \mathrm{S} .=11,5 \pm 1,02 ; \mathrm{n}=13$ ) para las del Andévalo, y entre 7 y $12(\bar{x} \pm$ E.S. $=9,3 \pm 0,8$; $\mathrm{n}=6$ ) para las de la Sierra Norte de Sevilla, pero esta diferencia entre ambas comarcas no es significativa. En las mieles de cosecha de apicultores de estas comarcas estudiadas anteriormente se observa un número medio de tipos por muestra ( $\bar{x} \pm$ E.S. $=12,5 \pm 0,68 ; n=23$ ) mayor que en las estudiadas en este trabajo $(\bar{x} \pm$ E.S. $=10,8 \pm 0,77 ; n=19)$ si bien esta diferencia no es significativa.

Los 39 tipos polínicos identificados pertenecen a 24 familias, 15 de las cuales están representadas por un sólo tipo, mientras que Asteraceae y Cistaceae lo están por cinco cada una. En el caso de Asteraceae, las frecuencias de los distintos tipos en cada muestra son bajas; mientras que en Cistaceae son elevadas en muchas de ellas.

Ninguno de los 39 tipos aparece en todas las muestras estudiadas, pero el polen de Echium plantagineum sólo está ausente en una de ellas y es el tipo dominante $(>45 \%)$ en varias. Asimismo, el polen de Cistus sp. (C. albidus, C. crispus), Cistus ladanifer, Cistus monspeliensis, Lavandula stoechas s.l. y Eucalyptus camaldulensis está presente en gran parte de las muestras, en algunos casos con porcentajes importantes. También el polen de Quercus sp. y Rubus ulmifolius aparece en más de la mitad de las mieles estudiadas. Por último, cabe señalar que el polen de Cistus libanotis, tipo Cytisus scoparius (Genisteae), Teucrium capitatum y Myrtus communis presenta una frecuencia considerable en alguna de las muestras, siendo la primera vez que el polen de Cistus libanotis y el de Teucrium capitatum aparecen en mieles de estas comarcas.

\section{DISCUSIÓN}

Nuestros resultados muestran que en todas las mieles ahora estudiadas el contenido de elementos indicadores de mielada es muy bajo con respecto al de granos de polen de plantas nectaríferas (índice HDE/P de Louveaux et al., 1978). De esto se deduce, de acuerdo con los criterios de Louveaux et al. (1978), que el néctar de flores es la principal materia prima de estas mieles y que la mielada tiene poca importancia. Esto contrasta con los resultados de los trabajos anteriores de estas comarcas (Ortiz, 1988; Ortiz y Fernández, 1992), en los que se estudiaron mieles de cosecha de apicultores (producidas durante todo el año) y se puso de manifiesto que la mielada es importante como fuente de miel, sobre todo en 


\begin{tabular}{|c|c|c|c|c|c|c|c|c|c|c|c|c|c|c|c|c|c|c|c|}
\hline \multirow{2}{*}{$\frac{\text { Localidades }}{\text { Muestras }}$} & \multirow[b]{2}{*}{1} & \multicolumn{4}{|c|}{ Niebla } & \multicolumn{4}{|c|}{ Alosno A } & \multicolumn{3}{|c|}{ Alosno B } & \multicolumn{4}{|c|}{ El Ronquillo } & \multicolumn{3}{|c|}{ El Pedroso } \\
\hline & & 2 & 3 & 4 & 1 & 2 & 3 & 4 & 5 & 1 & 2 & 3 & 4 & 1 & 2 & 3 & 1 & 2 & 3 \\
\hline T. Leucojum autumnale * & - & - & - & - & - & - & - & - & - & - & - & - & - & - & 1 & - & - & - & \\
\hline Apiaceae & - & - & - & - & 1 & - & - & - & - & 3 & 1 & - & - & - & - & - & - & - & - \\
\hline T. Bellis annua $*$ & 1 & - & 3 & 1 & - & - & - & - & - & 1 & - & 1 & - & - & - & - & - & - & - \\
\hline T. Carduus bourgeanus & - & - & - & - & - & 3 & 2 & 4 & 6 & 1 & 2 & - & 1 & - & - & - & - & - & 1 \\
\hline Centaurea sp. ${ }^{*}$ & - & - & - & - & - & - & - & - & - & - & 1 & - & - & - & - & - & - & - & - \\
\hline T. Crepis capillaris & - & - & - & 1 & - & 10 & 5 & 3 & 1 & - & - & 1 & - & - & - & - & - & - & - \\
\hline Helianthus annuus & - & - & - & - & - & - & - & - & - & - & - & - & - & - & 1 & - & - & - & - \\
\hline Echium plantagineum & 1 & - & 3 & 9 & 7 & 31 & 11 & 34 & 47 & 455 & 58 & 43 & 39 & 45 & 7 & 14 & 53 & 88 & 90 \\
\hline T. Raphanus raphanistrum & - & - & - & 2 & - & - & - & - & - & - & - & - & - & - & - & - & - & - & - \\
\hline Campanula erinus & 1 & - & - & 2 & 1 & 1 & - & 3 & - & - & - & - & - & - & - & - & 9 & - & - \\
\hline $\begin{array}{l}\text { Cistus sp. } \\
\text { (C. albidus, C. crispus) }\end{array}$ & - & - & - & - & 18 & 3 & 25 & 15 & 3 & 2 & 2 & 2 & 2 & 19 & 19 & 61 & 2 & - & 1 \\
\hline Cistus ladanifer & 30 & 40 & 28 & 12 & 4 & 10 & 7 & 12 & 4 & 11 & 4 & 34 & 5 & - & - & - & 2 & 4 & 2 \\
\hline Cistus libanotis* & - & - & 3 & 13 & - & - & - & - & - & - & - & - & - & - & - & - & - & - & - \\
\hline Cistus monspeliensis & 18 & 25 & 9 & 10 & 59 & 15 & 46 & 10 & 18 & 5 & 7 & 5 & 11 & - & - & - & - & - & . \\
\hline Cistus salvifolius & - & - & - & - & - & - & - & - & - & - & - & - & 3 & - & - & - & - & - & - \\
\hline Cyperus sp. ${ }^{*}$ & - & - & - & - & - & - & - & - & - & - & - & - & - & 3 & - & - & - & - & - \\
\hline Erica sp. & - & - & 1 & 1 & - & - & - & - & - & - & 1 & - & - & - & - & - & - & - & - \\
\hline Erica arbore & - & & 10 & - & - & - & - & - & - & - & - & - & - & - & - & - & - & - & \\
\hline T. Cytisus scoparius & - & - & - & - & - & - & - & - & - & - & - & - & 9 & 3 & 22 & 4 & - & 1 & - \\
\hline Ononis sp. & - & - & - & - & 7 & 1 & - & 5 & 8 & - & 1 & - & - & 4 & - & 1 & - & - & - \\
\hline Castanea sativa & - & - & - & - & - & - & - & - & - & - & - & - & - & - & - & - & 3 & - & 1 \\
\hline Quercus sp. & - & - & - & 1 & - & 3 & - & 5 & 1 & 1 & 1 & 1 & - & 4 & 11 & 1 & 2 & 2 & 1 \\
\hline Centaurium pulchellum * & - & - & - & - & - & - & - & 1 & - & 1 & - & - & - & - & - & - & - & - & - \\
\hline Lavandula stoechas s.l. & 20 & 5 & 4 & 14 & - & 18 & 3 & 1 & 7 & 2 & 2 & 3 & 3 & - & - & - & 3 & 1 & 2 \\
\hline Teucrium capitatum * & - & - & 1 & - & - & - & - & - & - & - & 1 & - & - & - & - & - & 12 & 2 & \\
\hline Eucalyptus camaldulensis & 26 & 27 & 38 & 32 & 1 & 1 & - & 2 & 1 & 2 & 10 & 5 & 11 & 14 & 18 & 10 & 3 & - & \\
\hline Myrtus communis & - & - & - & - & - & - & - & - & - & 14 & 2 & - & 1 & 2 & - & 1 & - & - & \\
\hline Olea europaea & 1 & - & - & - & - & - & - & 1 & - & 5 & 1 & 2 & 1 & - & 2 & - & - & - & \\
\hline Papaver sp. & - & - & - & - & - & - & - & - & - & - & - & - & - & 1 & 3 & 6 & - & - & \\
\hline Plantago sp. * & - & - & - & - & - & - & - & - & - & - & - & - & - & - & - & 1 & - & - & \\
\hline T. Festuca arundinacea $*$ & - & - & - & - & - & - & - & - & - & - & - & - & - & - & 7 & - & - & - & \\
\hline $\begin{array}{l}\text { T. Reseda luteola (R. luteola, } \\
\text { Sesamoides purpurascens) }\end{array}$ & & & & & & & & & & & & & & 1 & & & & & \\
\hline Fragar & - & $\begin{array}{l}- \\
-\end{array}$ & $\begin{array}{l}- \\
-\end{array}$ & - & & & - & $\begin{array}{l}- \\
-\end{array}$ & $\begin{array}{l}- \\
-\end{array}$ & - & $\overline{1}$ & - & 6 & 1 & - & - & - & - & \\
\hline ifolius & 1 & - & - & 1 & - & - & - & 1 & 1 & 3 & 2 & - & 4 & 2 & 3 & - & 9 & 1 & \\
\hline Citrus sp. ${ }^{*}$ & - & - & - & - & - & 1 & - & - & - & - & - & - & 1 & - & - & - & - & - & \\
\hline Anarrhinum bellidifolium * & - & - & - & - & - & 1 & - & 1 & 1 & - & - & - & - & - & - & - & - & - & \\
\hline Linaria sp. $*$ & - & - & - & - & - & - & - & - & - & - & - & 1 & - & - & - & - & - & - & \\
\hline Tamarix sp. & - & - & - & - & - & - & - & - & - & - & - & - & - & - & 1 & - & - & - & - \\
\hline Vitis vinifera * & - & - & - & - & - & - & - & - & - & 2 & - & 1 & - & - & - & - & - & - & - \\
\hline Otros (\#) & 2 & 2 & 1 & 2 & 4 & 3 & 2 & 2 & 3 & 2 & 3 & 3 & 2 & 2 & 5 & 2 & 2 & 2 & \\
\hline
\end{tabular}

Tabla 2.- Frecuencias de los distintos tipos polínicos en cada muestra; se presentan únicamente los porcentajes iguales o superiores a uno. *: Tipo no encontrado anteriormente en muestras de estas comarcas. (\#): Incluye varios tipos polínicos cuyos porcentajes individuales son inferiores a la unidad; algunos de estos tipos pueden estar incluídos entre los citados en la tabla. T: Tipo. Frequences of different pollen types in each sample; only percentages equal to or higher than one are indicated. *: Type not found before in samples from these regions. (\#): This includes several pollen types whose individual percentages are lower than one; some of them may be included among those indicated in the table. T: Type. 
la Sierra Norte de Sevilla. En la vecina comarca de Aracena se ha detectado un fenómeno similar, la mielada es importante en las mieles de cosecha de los apicultores y no lo es en mieles similares a las estudiadas en este trabajo (Ortiz et al., 1990). Aunque se ha puesto de manifiesto que los criterios melitopalinológicos no son concluyentes para definir las mieles de mielada y que en algunas de ellas el índice HDE/P es muy bajo (Serra et al. 1987), parece claro, por las observaciones comentadas, que en estas comarcas la mielada tiene poco significado como fuente de miel durante la primavera, pero no es así en otros periodos del año. Esta idea se ve reforzada por el reconocimiento de la encina (Quercus rotundifolia) como importante productora de mielada en este área durante los meses de Agosto a Octubre (Ortega, 1986).

En las muestras estudiadas en este trabajo, se ha encontrado un mayor número de tipos polínicos en las del Andévalo que en las de la Sierra Norte. Sin embargo en las mieles de cosecha de apicultores estudiadas anteriormente ocurre lo contrario (32 en El Andévalo frente a 54 en la Sierra Norte de Sevilla). Sin duda esto es debido a que en el trabajo actual más de dos tercio de las muestras estudiadas corresponden al Andévalo, mientras que en los anteriores el número de muestras de la Sierra Norte era casi el doble que el de las del Andévalo. Considerando todas las muestras estudiadas hasta ahora, el número de tipos correspondientes a la Sierra Norte es ostensiblemente mayor que el del Andévalo, lo cual concuerda con las apreciaciones de Talavera et al. (1988) de que el número de especies de interés apícola en la Sierra Norte es bastante superior al del Andévalo. Por otra parte en el conjunto de muestras estudiadas ahora se ha encontrado un número de tipos polínicos bastante menor al encontrado en las anteriores; es lo que cabia esperar, ya que las muestras actuales corresponden sólo a parte de la primavera y las anteriores al año completo.
A pesar de estas diferencias en número de tipos encontrados en los conjuntos de muestras de diferentes comarcas o diferentes periodos, el número medio de tipos por muestra no es significativamente distinto entre ambas comarcas, ni entre las muestras de primavera y las de todo el año. Es conocido el hecho de que, entre todas las fuentes de alimento disponibles, las abejas de una colonia utilizan en cada momento sólo unas pocas que le resultan más ventajosas (Visscher y Seeley, 1982; Ortiz, 1991). Por ello es lógico pensar que el número de fuentes utilizadas sea bajo si la oferta es poco diversa y que aumente, pero limitadamente, si la oferta se diversifica. La oferta florística primaveral en ambas comarcas es suficientemente diversa como para que las abejas diversifiquen su dieta al máximo, y produzcan unas mieles cuyos espectros son bastantes parecidos en cuanto al número de tipos. Probablemente las mieles producidas en otras estaciones presentarían espectros mucho menos diversos. En las mieles de todo el año están representados los tipos primaverales más importantes, más los de otras estaciones, y el número de tipos por muestra no aumenta significativamente porque a medida que se incorporan al espectro tipos importantes, el nivel de representación de los menos importantes disminuye por debajo del límite considerado (1\%). Sin duda la contaminación de la miel en el interior de la colmena con el polen de la cámara de cría manipulado por las abejas (Fernández y Ortiz, 1994) contribuye a diversificar el espectro de las mieles menos diversas, y tiende a homogeneizar el grado de diversidad de los espectros de las distintas muestras.

El que la familia Asteraceae esté representada por 5 tipos es un reflejo del gran número de sus taxones presentes en el territorio estudiado (Valdés et al., 1987b). En cambio, la buena representación del polen de Cistaceae indica la enorme apetencia de Apis mellifera por esta familia, representada en el área de 
estudio por alrededor de una veintena de especies (Valdés et al., 1987b). No obstante, tal como se señala en Ortiz (1994), aquí queda patente que el interés apícola de la familia se ciñe al género Cistus.

Apis mellifera obtiene néctar y polen de Echium plantagineum (Corbet y Delfosse, 1984), tendiendo este último a aparecer hiperrepresentado en los espectros polínicos de las mieles (Espada, 1984). Pérez et al. (1991), estudiando mieles de la vecina región de Extremadura, establecen un porcentaje mínimo de polen de viborera (Echium plantagineum) del $70 \%$ para considerar que una miel es monofloral de esta especie. De acuerdo con este criterio, dos de las mieles de la Sierra Norte (1/3 de las estudiadas) son monoflorales de viborera. Asimismo, una de las mieles de esta comarca estudiada anteriormente también sería de este tipo, si bien en el trabajo anterior no se hacía alusión a su carácter monofloral, y en otra el contenido de polen de viborera está muy próximo del citado límite. Aunque los trabajos anteriores habían puesto de manifiesto que Echium plantagineum es un recurso apícola importante en estas comarcas, los actuales resultados revalorizan el papel de esta especie como recurso nectarífero primaveral, y muestran que es especialmente interesante en la Sierra Norte, hasta el punto de permitir la producción de mieles monoflorales.

Aunque tradicionalmente se ha considerado que las especies de Cistus no producen néctar (Rita, 1983; Salas, 1984, p. e.), recientemente se detectó producción de cantidades no cuantificables en varias especiẹs de Cistus (Herrera, 1985; Brandt y Gottsberger, 1988; Talavera et al., 1988) y más tarde se ha comprobado que todas las especies de Cistus presentes en Andalucía Occidental producen cantidades considerables de néctar por flor (Talavera et al., 1993; Ortiz, 1994). Sin embargo, también se ha observado que en Andalucía Occidental las abejas visitan las flores de las jaras (Cistus sp.) principalmente para recoger su polen (Ortiz, 1994). Dado que los granos de polen de Cistus sp. son de gran tamaño en relación con los otros tipos encontrados (véase Valdés et al., 1987a), el valor de las jaras como recursos polínicos es mucho mayor que el que reflejarían directamente las frecuencias de aparición de su polen en los espectros polínicos (Buchmann y O'Rourke, 1991; Biesmeijer et al., 1992). Si bien en trabajos anteriores se había destacado el papel de las especies de Cistus como importantes recursos polínicos en estas comarcas, los actuales resultados lo resaltan aún más y muestran claramente que las especies de Cistus son las principales fuentes de polen primaverales en estas áreas, que equivale a decir que lo son en todo el año ya que en nuestro territorio las abejas recogen la mayor parte del polen en esta estación (Ortiz, 1991). En la Sierra Norte destacan Cistus crispus y $C$. ladanifer; en el Andévalo Cistus ladanifer y $C$. monspeliensis principalmente, aunque también Cistus crispus y en las zonas más próximas al litoral Cistus libanotis (véase su área de distribución en Valdés et al., 1987b). En ambas comarcas hay extensos jarales de los que se pueden obtener importantes cosechas de polen (Talavera et al., 1988).

Es sabido que el polen de Labiadas se encuentra infrarrepresentado en los espectros polínicos de las mieles (Stanley y Linskens, 1974; Serra et al., 1986, 1987). Gómez et al. (1994), estudiando mieles de la vecina región portuguesa del Bajo Alentejo, establecen que un $15 \%$ de polen de cantueso (Lavandula stoechas) es suficiente para considerar a una miel monofloral de esta especie; Pérez et al. (1991) bajan este límite al 9\% en mieles extremeñas. La presencia del polen de Lavandula stoechas s.l. en muchas de las muestras de estas comarcas estudiadas anteriormente, aunque en porcentajes bajos, mostraba que el cantueso es un recurso nectarífero importante en el área. Los actuales 
resultados revalorizan la importancia concedida a este recurso y muestran que, al menos en El Andévalo, es posible la obtención de mieles monoflorales de cantueso en primavera. El hecho de que el polen de Teucrium capitatum no hubiese aparecido en ninguna de las muestras anteriores y que se encuentre en varias de las actuales, en una de ellas con una frecuencia muy considerable, indica que éste es un recurso nectarífero secundario en la globalidad del área pero localmente importante.

El papel apícola de Eucalyptus sp., Quercus sp., Rubus ulmifolius, Genisteae y Myrtus communis, cuyo polen se encuentra bien representado en estas muestras, ya ha sido puesto de manifiesto en trabajos anteriores. Aunque los principales recursos apícolas de estas comarcas ya habían sido puestos de manifiesto en los trabajos previos, el presente estudio ha permitido conocer otros de interés más local o secundario, y sobre todo establecer una valoración más precisa de los primeros, manifestándose la posibilidad de obtención de mieles monoflorales de viborera y cantueso durante la primavera.

AGRADECIMIENTOS. Los autores desean expresar su agradecimiento a D. F. J. García Ugidos por facilitar el material estudiado y al Prof. S. Talavera y a la Dra. M. J. Díez por su ayuda y consejos. El presente trabajo ha sido financiado con cargo al proyecto de la DGICYT PA 85-297.

\section{BIBLIOGRAFÍA}

ARROYO, J. -1988- Fenología de la floración en especies del matorral del sur de España. Lagascalia, 15 (Extra): 593-606.

ARROYO, J. -1990- Spatial variation of flowering phenology in the mediterranean shrublands of southern Spain. Isr. J. Bot., 39: 249-262.

BIESMEIJER, J.C., B. VAN MARWIJK, K. VAN DEURSEN, W. PUNT \& M.J. SOMMEIJER 1992- Pollen sources for Apis mellifera L. (Hym, Apidae) in Surinam, based on pollen grain volume estimates. Apidologie, 23: 245-256.

BRANDT, U. \& G. GOTTSBERGER -1988- Flower phenology, pollinating insects and breeding systems in Cistus, Halimium and Tuberaria species in Portugal. Lagascalia, 15 (Extra): 625-634.

BUCHMANN, S.L. \& M.K. O'ROURKE -1991Importance of pollen grain volumes for calculating bee diets. Grana, 30: 591-595.

CORBET, S.A. \& E.S. DELFOSSE -1984Honeybees and the nectar of Echium plantagineum L. in south-eastern Australia. Austral. J. Ecol., 9: 125-139.

ERDTMAN, G. -1960- The acetolysis method. A revised description. Svensk. Bot. Tidskr., 54: 561-564.

ESPADA, T. -1984- Contribución al conocimiento de las mieles de producción nacional: espectro polínico de la miel de Brezo de Cataluña. Vida Apícola, 11: 17-20.

FERNÁNDEZ, I. \& P.L. ORTIZ -1994- Pollen contamination of honey by bees inside the hive. Grana, 33: 282-285.

GÓMEZ, C. y C. SÁENZ -1985- Estudio del sedimento polínico de las mieles de la reserva biológica de Doñana (Huelva) España. Anales Asoc. Palinol. Lengua Esp., 2: 369-374.

GÓMEZ, C., J. PAIVA y M.T. LEITAO -1994Espectro polínico de mieles del Baixo Alentejo I, región de Almodovar (Portugal). En I. LASERNA (ed.) Polen y esporas: contribución a su conocimiento. Ed. Universidad de La Laguna. Tenerife: 253-258.

HERRERA, J. -1985- Nectar secretion patterns in southern Spanish mediterranean shrublands. Israel J. Bot., 34: 47-58.

LADERO, M. -1987- La España LusoExtremadurense. En M. PEINADO LORCA \& S. RIVAS-MARTÍNEZ (eds.) La vegetación de España. Universidad de Alcalá de Henares. Alcalá de Henares: 453-486.

LOUVEAUX, J., A. MAURIZIO \& G. VORWOHL -1978- Methods of Melissopalynology. Bee World, 59: 139-157.

MAURIZIO, A. -1979- Microscopy of honey. En E. CRANE (ed.) Honey. A comprehensive survey. Heinemann. London: 240-257.

ORTEGA, J.L. -1986- Flora de interés apícola de la España Peninsular. Actas II Congr. Nac. Apic. (Gijón, 1984): 156-171. 
ORTIZ, P.L. -1988- Estudio melitopalinológico en El Andévalo (Huelva). Anales Asoc. Palinol. Lengua Esp., 4: 64-72.

ORTIZ, P.L. -1991- Melitopalinología en Andalucía Occidental. Tesis Doctoral. Editorial Universidad de Sevilla. Sevilla.

ORTIZ, P.L. -1994- The Cistaceae as food resources for honey bees in SW Spain. J. Apic. Res., 33 (3): 136-144.

ORTIZ, P.L. e I. FERNÁNDEZ -1992- Estudio microscópico de miel y polen apícola de la provincia de Sevilla. Acta Bot. Malacitana, 17: 183-193.

ORTIZ, P.L., I. FERNÁNDEZ y M. MARTIN CACAO -1990- Estudio melitopalinológico en la comarca de Aracena (Huelva). Lagascalia, 16: $61-76$.

PÉREZ, C., P L. RODRÍGUEZ \& J.L. RODRÍGUEZ -1991- Estudio polínico de algunas mieles monoflorales de Extremadura. Actas IV Congr. Nac. Apic. (Zaragoza, 1988): 95-103.

RITA, J. -1983- Flora melífera de la provincia de Lleida. Ed. Ecxma. Diputación de Lleida. Lleida.

SALA, A. -1984- Plantas melíferas de la zona de Jijona (Alicante). Vida Apícola, 11: 52-56.

SERRA, J. \& S. CAÑAS -1988- Caractteristiche fisico-chimique, composizione e spectro pollinico del miele di eucalipto (Eucalyptus spp.) prodotto in Spagna. Apicoltura, 4: 59-81.

SERRA, J., A. GÓMEZ y J. GONELL -1986- Estudio del espectro polínico de la miel de espliego (Lavandula latifolia Mod. con Lavandula pedunculata Cav.) producida en Cuenca, Guadalajara y Soria. Actas II Congr. Nac. Apic. (Gijón, 1984): 137-144.

SERRA, J., A. GÓMEZ y J. GONELL -1987Composición, propiedades fisico-químicas y espectro polínico de algunas mieles monoflorales de España. Alimentaria, XXIV (185): 61-84.

STANLEY, R.G. \& H.F. LINSKENS -1974-Pollen. Biology Biochemistry Management. SpringerVerlag. Berlin, Heidelberg, New York.

TALAVERA, S., J. HERRERA, J. ARROYO, P.L. ORTIZ y J.A. DEVESA -1988- Estudio de la flora apícola de Andalucía Occidental. Lagascalia, 15 (Extra): 567-591.

TALAVERA, S., P.E. GIBBS \& J. HERRERA 1993-Reproductive biology of Cistus ladanifer L. (Cistaceae). Plant Syst. Evol., 186: 123-134.
TELLO, E. -1982- La miel de Aracena. Arch. Zootecnia, 31: 293-303.

VALDÉS, B., M.J. DÍEZ e I. FERNÁNDEZ -1987aAtlas polínico de Andalucía Occidental. Inst. Des. Regional y Ecxma. Diputación de Cádiz. Sevilla.

VALDÉS, B., S. TALA VERA y E. FERNÁNDEZGALIANO -1987b- Flora vascular de Andalucía Occidental. 3 vols. Ketres. Barcelona.

VERGERON, P. -1964- Interprétation statistique des résultats en matière d'analyse pollinique des miels. Ann. Abeille, 7: 349-364.

VISSCHER, P.K. \& T.D. SEELEY -1982- Foraging strategy of honeybee colonies in a temperate deciduous forest. Ecology, 63: 1790-1801.

Aceptado para su publicación en Mayo de 1995

Departamento de Biología Vegetal y Ecología. Universidad de Sevilla. Apdo. 1095. 41080 Sevilla. 